\title{
Régénération in vitro à partir de cals d'embryons immatures de maïs. Aspects quantitatifs et trans- mission héréditaire
}

\author{
Roland BRUNEAU
}

I.N.R.A., Station d'Amélioration des Plantes, B. V. 1540, F 21034 Dijon Cedex

RÉSUMÉ

\begin{abstract}
A partir de plusieurs génotypes de Zea mays, des cals issus d'embryons immatures ont été cultivés in vitro pour étudier quantitativement la régénération de plantes suivant deux critères : la proportion d'embryons morphogènes et le rendement en plantes de ceux-ci.

L'obtention d'embryons morphogènes est très dépendante du génotype ; cette propriété se transmet par croisement. La durée de culture primaire ne semble pas déterminante ni le type de régulateur de croissance employé $(2,4 \mathrm{D}$ et $\mathrm{pCA})$. On note toutefois un effet de la plante-mère.

La quantité de plantes régénérées paraît aussi liée au génotype ; les embryons de taille comprise entre 1 et $2,15 \mathrm{~mm}$ sont plus prolifiques pour l'ensemble des génotypes testés.

Les fréquences de rendement en plantes sont très variables. Les plantes régénérées repiquées en serre ont présenté une réduction de la taille et du nombre de feuilles. Le nombre chromosomique sur les plantes contrôlées est $2 \mathrm{n}=20$.
\end{abstract}

Mots clés additionnels : Zea mays. through crosses.

Immature embryos from several Zea mays lines were grown in vitro in order to study plant regeneration rate quantitatively according to two criteria : the proportion of morphogenous embryos and the yield of plants from them. The proportion of morphogenous embryos was strongly dependent on genotype ; this property was cross-transmissible. Neither the duration of primary culture nor the growth regulators used $(2,4-\mathrm{D}$ or $\mathrm{pCA})$ seemed to have a determining effect. However, a mother plant effect was observed. The number of regenerated plants also seemed linked to genotype ; for all tested lines, embryos between 1 and $2.15 \mathrm{~mm}$ were more prolific. Plant yield rates were very variable. On regenerated plants transplanted in the greenhouse, a decrease in size and leaf number was observed. All cytologically checked individuals showed 20 chromosomes.

Additional key words : Zea mays.

\section{INTRODUCTION}

L'obtention de plantes néoformées a été réalisée chez plusieurs Monocotylédones en partant de divers explants (apex, microspores, fragments de tiges).

C'est en utilisant les embryons immatures que les meilleurs résultats semblent avoir été obtenus chez les Graminées comme l'avoine (CUMMINGS et al., 1976), le sorgho (GAMBORG et al., 1977) et l'orge (DALE \& DeAmbrogio, 1979). Chez le maïs, GreEN (1982) obtient une embryogenèse somatique et la régénéra- tion de plantes à partir de cals friables d'embryons immatures ou de suspensions cellulaires.

Cependant, la culture d'embryons immatures consistant en une callogenèse sur un milieu à forte concentration en $2,4 \mathrm{D}$, puis un repiquage sur un milieu à faible teneur de la même auxine, telle que l'ont décrite GREen \& PHILlips (1975), semble à ce jour la technique la plus aisée pour régénérer in vitro des plantes entières de maïs. A la suite de ces travaux et sur le même type d'explant, des résultats surtout qualitatifs concernant les possibilités de plusieurs génotypes à la 
callogenèse et à la régénération ont été publiés par GREEN (1979) et BECKET \& POLlaCSEK (1979).

L'aspect quantitatif de la néoformation de plantes chez le maïs à partir d'embryons immatures a été particulièrement étudié par BECKERT (1982) mais en utilisant une seule lignée.

Complémentairement à ces travaux, notre objectif a donc été de préciser les potentialités in vitro à la régénération, de matériels de différentes origines génétiques (lignées, croisements), tout en approfondissant certains aspects (régulateur de croissance, durée de culture préliminaire).

\section{MATÉRIEL ET MÉTHODES}

\section{A. Lignées}

Les 7 lignées employées ont différentes origines génétiques (tabl. 1). Les graines proviennent de la Station I.N.R.A. d'Amélioration des Plantes de ClermontFerrand.

\section{TABLEAU 1}

Origine des génotypes utilisés pour la culture d'embryons immatures.

Origin of genotypes used for immature embryo cultures.

\begin{tabular}{|c|c|c|c|}
\hline Groupe & Génotype & $\begin{array}{l}\text { Indice de } \\
\text { précocité } \\
\left.\qquad{ }^{*}\right)\end{array}$ & Origine \\
\hline \multirow{4}{*}{$\begin{array}{l}\text { Groupe } \\
\text { américain }\end{array}$} & $\mathrm{CO158}$ & -10 & Confidentielle. \\
\hline & A1 88 & 0 & $\begin{array}{l}\text { Sélection dans l'hybride «Sil- } \\
\text { ver King } \times \text { « M. W. Dant } \text {. }\end{array}$ \\
\hline & A631 & & \\
\hline & A641 & +3 & $\begin{array}{l}\text { Issu du croisement «N.D. } \\
203 » \times \ll \text { B } 14 » .\end{array}$ \\
\hline \multirow{3}{*}{$\begin{array}{l}\text { Groupe } \\
\text { européen }\end{array}$} & F19 & 0 & $\begin{array}{l}\text { Sélection entre des populations } \\
\text { européennes, croisées par des } \\
\text { hybrides nord-américains. }\end{array}$ \\
\hline & F1048 & +1 & $\begin{array}{l}\text { Issu du croisement «F115» } \times \\
\text { «F22». }\end{array}$ \\
\hline & F1412 & +2 & $\begin{array}{l}\text { Issu du croisement «F52 » } \\
\text { «W135R ». }\end{array}$ \\
\hline
\end{tabular}

(*) Décalages de floraison $q$ en jours par rapport à la lignée témoin « W182E » (I.N.R.A., Clermont-Ferrand).

\section{B. Conditions de culture des plantes-mères}

Les plantes-mères sur lesquelles seront prélevés les embryons immatures sont élevées en serre ou en chambre climatisée. Dans le $1^{\text {er }}$ cas, elles sont, durant l'hiver, maintenues à la température de $18^{\circ} \mathrm{C}$ environ ; un appoint d'éclairage est apporté par des lampes à incandescence durant $16 \mathrm{~h} /$ jour.

Dans le $2^{\mathfrak{e}}$ cas, la température ambiante est de $20 \pm$ $2{ }^{\circ} \mathrm{C}$; l'énergie lumineuse (tubes fluorescents et lampes à incandescences) reçue par les plantes est d'environ $25 \mathrm{~W} / \mathrm{m}^{2}$ durant $16 \mathrm{~h} /$ jour.

\section{Prélèvement des embryons}

Après enlèvement des spathes, les épis, issus de plusieurs plantes, sont stérilisés durant $20 \mathrm{mn}$ dans une solution d'hypochlorite de calcium à 2,5 p. 100 et rincés 2 fois à l'eau ultra pure stérile.

Les embryons prélevés stérilement sur toute la surface de l'épi sous une hotte à flux laminaire sont mesurés sur la plus grande longueur du scutellum ; ils ont, suivant les expériences, une taille comprise entre 1 et $4 \mathrm{~mm}$ environ ou entre 2 et $3 \mathrm{~mm}$ environ. Dans ce dernier intervalle, le temps écoulé de la fécondation au prélèvement est de 15 à $29 \mathrm{j}$.

Chaque explant est posé sur le milieu gélosé, axe embryonnaire à son contact.

Toutes les cultures in vitro se font en tubes de verre $24 \mathrm{~mm}$ de diamètre et $150 \mathrm{~mm}$ de hauteur.

\section{Milieux de culture}

Le milieu de base est celui utilisé par GREEN \& PHILliPs en 1975. Pour obtenir la régénération de plantes, 2 auxines ont été employées, le 2,4D et le pCA.

Avec le 2,4D (2 mg/l), la culture primaire dure entre 10 et $20 \mathrm{j}$, moins dans certaines expériences ; les cals développés sont ensuite transférés, pour $25 \mathrm{j}$, sur un milieu contenant $0,25 \mathrm{mg} / 1$ de $2,4 \mathrm{D}$, puis repiqués mensuellement jusqu'à extinction de la production de plantes sur le milieu de base sans auxine.

Dans le cas d'utilisation du pCA ( $2 \mathrm{mg} / \mathrm{l})$, la durée de culture primaire est comprise entre 15 et $25 \mathrm{j}$, les repiquages suivants s'effectuant tous sur le milieu de base.

Tous les milieux sont autoclavés durant $20 \mathrm{mn}$ à $110-112^{\circ} \mathrm{C}$.

\section{E. Conditions de culture in vitro}

La chambre de culture est maintenue à une température diurne de $25^{\circ} \mathrm{C}$ et à une température nocturne de $22 \pm 2{ }^{\circ} \mathrm{C}$. L'éclairage des cultures est assuré par des tubes fluorescents «cool white» fournissant $4,6 \mathrm{~W} / \mathrm{m}^{2}$ environ au niveau du matériel végétal.

\section{F. Conditions de culture des plantes régénérées}

Lorsque les plantes ont des racines suffisamment développées, elles sont transférées en chambre climatisée (16 h de lumière, $20^{\circ} \mathrm{C}$ ) et laissées $8 \mathrm{j}$ le capuchon du tube de culture enlevé.

Au terme de cette période, elles sont repiquées en pot sur un mélange tamisé sable-terre et arrosées avec une solution nutritive minérale. Après un séjour de 3 semaines, en chambre climatisée, les plantes sont transférées en serre.

\section{G. Protocole expérimental}

Pour l'ensemble des manipulations, 2 critères sont étudiés : le $1^{\text {er }}$ est le nombre d'embryons donnant une plante au moins, sur le nombre total d'embryons mis en culture, exprimé en p. 100 de régénération; le $2^{\mathrm{e}}$ critère est le nombre de plantes régénérées par scutellum embryogène (rendement en plantes).

Pour évaluer à un stade précoce les potentialités de la productivité des embryons en plantes, celles-ci sont comptabilisées in vitro lorsqu'elles mesurent au moins $1 \mathrm{~cm}$ de hauteur avec 2 feuilles formées. Dans toutes les expériences, sauf celle décrite au paragraphe A, 3 
suivant, les lots d'embryons ne sont pas individualisés par plante.

\section{RÉSULTATS}

\section{A. Facteurs influençant la proportion d'embryons morphogènes}

\section{Importance du régulateur de croissance}

Pour comparer leur propriété morphogène, 2 régulateurs de croissance ont été testés sur 6 lignées, avec des embryons dont la taille est comprise entre 1 et $4 \mathrm{~mm}$ environ ( $\mathrm{tabl} .2$ ).

Aucun effet spécifique des substances de croissance n'est mis en évidence ; $2,4 \mathrm{D}$ et pCA ont donc ici des propriétés morphogènes comparables.

\section{TABLEAU 2}

Comparaison de la proportion d'embryons morphogènes suivant le type de régulateur de croissance. La lignée «F1412 » n'est pas incluse dans ce test.

Comparison of proportion of morphogenous embryos according to the growth regulator. Line 'F1412' not included in test.

\begin{tabular}{lcccc}
\hline \multicolumn{1}{c}{ Auxine } & \multicolumn{2}{c}{$2,4 \mathrm{D}$} & \multicolumn{2}{c}{$\mathrm{pCA}$} \\
\hline Lignée & Nombre $\left({ }^{1}\right)$ & Total $\left({ }^{2}\right)$ & Nombre $\left({ }^{\mathrm{l}}\right)$ & Total ${ }^{2}{ }^{2}$ \\
\hline A188 & 42 & 108 & 57 & 108 \\
A641 & 24 & 108 & 30 & 108 \\
F19 & 17 & 94 & 15 & 96 \\
CO158 & 20 & 107 & 12 & 102 \\
F1048 & 11 & 108 & 15 & 107 \\
A631 & 5 & 90 & 6 & 89 \\
Totaux & 119 & 615 & 135 & 610 \\
\multicolumn{3}{c}{$\chi^{2}=1,46$ N.S. } \\
\hline \hline
\end{tabular}

$\left({ }^{1}\right)$ Nombre d'embryons ayant donné au moins une plante.

( ${ }^{2}$ Total des embryons mis en culture.

N.S. Résultat non significatif.

\section{Effet génotypique}

Avec les mêmes données, sans tenir compte du type de régulateur de croissance, un test d'homogénéité incluant la lignée « F1412 » dont un seul embryon a régénéré sur 209 mis en culture est hautement significatif $\left(\chi_{6}^{2}=189,07\right)$. Le classement décroissant des lignées correspond à celui du tableau 2 ; le groupe constitué de «F19», «CO158 » et «F1048 » est assez homogène.

\section{Influence de la durée de culture primaire}

Avec la meilleure lignée embryogène, «A188», l'influence de la durée de culture préliminaire sur un milieu 2,4D riche en auxine a été étudiée. Des embryons d'une longueur de 2 à $3 \mathrm{~mm}$ environ ont été prélevés sur des plantes différentes élevées dans des conditions identiques (date de semis, intervalle fécondation-prélèvement, jour de mise en culture).

Deux expériences successives pour des durées minimales de culture de $24 \mathrm{~h}$ et $6 \mathrm{~h}$ aboutissent à la même conclusion: aucun effet du traitement. Par contre, l'effet de la plante-mère est hautement significatif (tabl. 3a, b, c, d).
TABLEAU 3

Proportion d'embryons morphogènes en fonction de la culture primaire sur milieu contenant $2 \mathrm{mg} / \mathrm{l}$ de $2,4 \mathrm{D}$.

Proportion of morphogenous embryos for primary culture on a medium with $2 \mathrm{mg} / \mathrm{l}$ of $2,4-D$.

a) Durées de 1 jour à 3 jours.

Duration from 1 to 3 days.

\begin{tabular}{ccccccc}
\hline $\begin{array}{c}\text { Lignée A188 } \\
\text { embryons de } \\
2 \text { à 3 mm }\end{array}$ & \multicolumn{5}{c}{ Durée de culture préliminaire } \\
\cline { 2 - 7 } $\mathrm{n}^{\circ}$ des plantes & 1 & $\frac{9}{20}\left(^{*}\right)$ & $\frac{3}{20}$ & $\frac{5}{20}$ & $\frac{1}{19}$ & $\frac{18}{79}$ \\
$(1$ épi par plante) & 2 & $\frac{16}{20}$ & $\frac{16}{20}$ & $\frac{14}{20}$ & $\frac{14}{20}$ & $\frac{60}{80}$ \\
\hline Totaux & 3 & $\frac{13}{19}$ & $\frac{15}{20}$ & $\frac{6}{17}$ & $\frac{8}{19}$ & $\frac{42}{75}$ \\
\hline \hline
\end{tabular}

(*) Nombre d'embryons morphogènes sur le nombre total d'embryons mis en culture. Number of morphogenous embryos/number of embryos cultured.

b) Analyse de variance sur les données transformées ( $\sqrt{\operatorname{arc}} \sin )$.

Variance analysis of transformed data ( $\sqrt{\operatorname{arc}} \sin )$.

\begin{tabular}{lccrr}
\hline $\begin{array}{c}\text { Source de } \\
\text { variation }\end{array}$ & $\begin{array}{c}\text { Somme des } \\
\text { carrés }\end{array}$ & $\begin{array}{c}\text { Degré de } \\
\text { liberté }\end{array}$ & $\begin{array}{c}\text { Carré } \\
\text { moyen }\end{array}$ & F \\
\hline Totale & 3127,05 & 11 & & \\
Plantes & 2243,30 & 2 & 1121,65 & $18,49^{* *}$ \\
Durées & 519,75 & 3 & 173,25 & 2,86 N.S. \\
Erreur & 364,00 & 6 & 60,67 & \\
\hline \hline
\end{tabular}

** significatif au niveau $1 \%$.

c) Durées de $6 h$ à 2 jours.

Duration from $6 \mathrm{~h}$ to 2 days.

\begin{tabular}{lllllllll}
\hline \hline $\begin{array}{c}\text { Lignée A188 } \\
\text { embryons de } \\
2 \text { à 3 mm }\end{array}$ & \multicolumn{6}{c}{ Durée de culture préliminaire } \\
\cline { 2 - 8 } & & $6 \mathrm{~h}$ & $12 \mathrm{~h}$ & $24 \mathrm{~h}$ & $48 \mathrm{j}$ & $10 \mathrm{j}$ & Totaux \\
\hline $\mathrm{n}^{\circ}$ des plantes & 14 & $\frac{14}{16}$ & $\frac{10}{18}$ & $\frac{10}{18}$ & $\frac{10}{18}$ & $\frac{7}{18}$ & $\frac{31}{88}$ \\
(1 épi par plante) & 15 & $\frac{4}{19}$ & $\frac{1}{20}$ & $\frac{1}{18}$ & $\frac{0}{20}$ & $\frac{1}{12}$ & $\frac{7}{89}$ \\
\hline Totaux & & $\frac{18}{35}$ & $\frac{11}{38}$ & $\frac{11}{36}$ & $\frac{10}{38}$ & $\frac{8}{30}$ & \\
\hline \hline
\end{tabular}

Transformation des données et calculs semblables aux durées 1 à 3 jours.

d) Analyse de variance.

Variance analysis.

\begin{tabular}{lccrr}
\hline $\begin{array}{c}\text { Source de } \\
\text { variation }\end{array}$ & $\begin{array}{c}\text { Somme des } \\
\text { carrés }\end{array}$ & $\begin{array}{c}\text { Degré de } \\
\text { liberté }\end{array}$ & $\begin{array}{c}\text { Carré } \\
\text { moyen }\end{array}$ & F \\
\hline Totale & 4198,62 & 9 & & \\
Plantes & 3305,13 & 1 & 3305,13 & $68,16^{* *}$ \\
Durées & 699,51 & 4 & 174,88 & 3,61 N.S. \\
Erreur & 193,98 & 4 & 48,49 & \\
\hline
\end{tabular}




\section{Transmission de la capacité à régénérer}

Les lignées «A188», «CO158》 et «F1412» ont été retenues pour leur capacité morphogene respectivement élevée, moyenne et faible. Des croisements réciproques ont été réalisés et comparés aux parents autofécondés (tabl $4 a$ et b). En F1, il y a toujours eu néoformation quel que soit le sens du croise-

\section{TABLEAU 4}

Transmission des capacités de régénération des embryons par croisement.

Transmission of embryo regeneration ability by crossing.

a) Lignées « AI88》 et «F1412》.

\begin{tabular}{|c|c|c|c|c|}
\hline $\begin{array}{l}\text { Autofécondation } \\
\text { ou } \\
\text { croisement }\end{array}$ & A188 AF & $\begin{array}{l}\mathrm{A} 188 \\
\times \\
\mathrm{F} 1412\end{array}$ & $\begin{array}{l}\text { F1412 } \\
\times \\
\text { A } 188\end{array}$ & F1412 AF \\
\hline $\begin{array}{l}\text { Nombre d'embryons } \\
\text { ayant donné une plante } \\
\text { au moins }\end{array}$ & 17 & 83 & 97 & 0 \\
\hline $\begin{array}{l}\text { Nombre total } \\
\text { d'embryons en culture }\end{array}$ & 94 & 189 & 185 & 192 \\
\hline$\%$ de régénération & 18 & 43,9 & 52,4 & 0 \\
\hline $\begin{array}{l}\text { Comparaison des hybri- } \\
\text { des réciproques }\end{array}$ & \multicolumn{4}{|c|}{$\chi_{1}^{2}=2,71$ N.S. } \\
\hline $\begin{array}{l}\text { Comparaison hybrides/ } \\
\text { parents }\end{array}$ & \multicolumn{4}{|c|}{$\chi_{1}^{2}=28,71^{* *}$} \\
\hline
\end{tabular}

b) Lignées « COIS8 » et « F1412》.

\begin{tabular}{|c|c|c|c|c|}
\hline $\begin{array}{c}\text { Autofécondation } \\
\text { ou } \\
\text { croisement }\end{array}$ & CO158 AF & $\begin{array}{l}\mathrm{CO} 158 \\
\times \\
\mathrm{F} 1412\end{array}$ & $\begin{array}{l}\mathrm{F} 1412 \\
\times \\
\mathrm{CO} 158\end{array}$ & F1412 AF \\
\hline $\begin{array}{l}\text { Nombre d'embryons } \\
\text { ayant donné une plante } \\
\text { au moins }\end{array}$ & 59 & 16 & 21 & 0 \\
\hline $\begin{array}{l}\text { Nombre total } \\
\text { d'embryons en culture }\end{array}$ & 192 & 192 & 196 & 192 \\
\hline$\%$ de régénération & 30,7 & 8,3 & 10,7 & 0 \\
\hline $\begin{array}{l}\text { Comparaison des hybri } \\
\text { des réciproques }\end{array}$ & \multicolumn{4}{|c|}{$\chi_{1}^{2}=0,63 \mathrm{~N} . \mathrm{S}}$. \\
\hline $\begin{array}{l}\text { Comparaison hybrides } \\
\text { parent }\end{array}$ & \multicolumn{4}{|c|}{$\chi_{1}^{2}=41,76^{* *}$} \\
\hline
\end{tabular}

ment; les hybrides réciproques sont équivalents $\left(\chi^{2}\right.$ N.S.).

La lignée «A188》 régénère moins que ses hybrides, le cas est inverse avec « CO158».

\section{B. Facteurs influençant le rendement en plantes des embryons morphogènes}

\section{Nombre moyen de plantes par lignée}

L'ensemble des plantes comptabilisées provient d'embryons ou de fractions d'embryons repiqués jusqu'à extinction de leur propriété morphogène.

Le tableau 5 donne les rendements en plantes pour chaque lignée ; le nombre moyen de plantes par rapport au total des embryons en culture est faible ; le rendement rapporté aux seuls embryons morphogènes montre que «A188 » est la lignée la plus productive ; vient ensuite un groupe constitué de "A641», «F19 » et «CO158», puis, à rendement beaucoup plus faible, «F1048» et «A631 ». Cet ordre est proche de celui obtenu pour la régénération de plantes («A188», «A641 », groupe «F19»-«CO158»-«F1048», puis «A631»).

\section{Rendement en plantes par classe de taille}

Le tableau 6 regroupe, pour les 7 lignées et par classe de taille, le total des plantes obtenues avec le

\section{TABLEAU 6}

Rendement en plantes des embryons de 1 à $4 \mathrm{~mm}$ par classe de taille (lignées A188, A641, F19, CO158, F1048, A631, F1412).

Plant yield for embryos ranging from 1 to $4 \mathrm{~mm}$ (lines A188, A64I, F19, CO158, F1048, A631 and F1412).

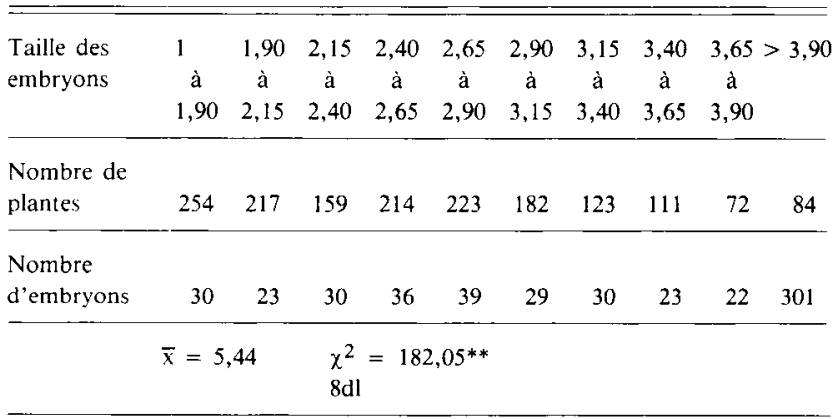

Nombre moyen

de plantes par

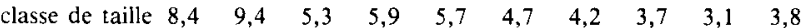

TABLEAU 5

Rendement en plantes des embryons de 1 à $4 \mathrm{~mm}$.

Plant yield from $1-4 \mathrm{~mm}$ embryos.

\begin{tabular}{|c|c|c|c|c|c|}
\hline Lignées & $\begin{array}{l}\text { Nombre total } \\
\text { d'embryons } \\
\text { en culture }\end{array}$ & $\begin{array}{c}\text { Nombre } \\
\text { d'embryons } \\
\text { morphogènes }\end{array}$ & $\begin{array}{c}\text { Nombre total } \\
\text { de plantes } \\
\text { obtenues }\end{array}$ & $\begin{array}{c}\text { Nombre moyen de } \\
\text { plantes par rapport } \\
\text { au total } \\
\text { des embryons }\end{array}$ & $\begin{array}{l}\text { Nombre moyen de } \\
\text { plantes par } \\
\text { embryon morphogène }\end{array}$ \\
\hline A188 & 407 & 130 & 909 & 2,2 & 7 \\
\hline A641 & 216 & 54 & 241 & 1,1 & 4,5 \\
\hline F19 & 190 & 32 & 156 & 0,8 & 4,9 \\
\hline CO158 & 398 & 47 & 263 & 0,7 & 4,9 \\
\hline F1048 & 215 & 26 & 49 & 0,2 & 2,0 \\
\hline A631 & 179 & 11 & 18 & 0,1 & 1,6 \\
\hline F1412 & 624 & 1 & 3 & - & 3 \\
\hline
\end{tabular}


nombre d'embryons morphogènes correspondants ; le $\chi^{2}$ d'homogénéité calculé est hautement significatif. Cela provient principalement des classes de taille regroupées (1 à $1,90 \mathrm{~mm}$ ) et de la classe 1,90 à $2,15 \mathrm{~mm}$; les tailles d'embryons comprises dans ces valeurs produisent, en moyenne, plus de plantes.

\section{Fréquences des plantes néoformées}

A partir du nombre de plantes néoformées par chaque embryon, on a établi des fréquences de rendement (nombre d'embryons ayant donné 0 plante, 1 plante, 2 plantes, etc...), pour des embryons de 1 à $4 \mathrm{~mm}$ environ (lignées «A 188 », «A641», «F19», «CO158») et des embryons de 2 à $3 \mathrm{~mm}$ environ (lignées «A188», «CO158», hybrides réciproques «A188» X «F1412», «CO158» X F1412»).

La répartition des fréquences de rendement en plantes par embryon a été comparée à la distribution théorique de la loi de Poisson ; tous les tests $\chi^{2}$ sont hautement significatifs. Les observations réalisées ne peuvent donc être assimilées à une loi de Poisson ; les écarts proviennent surtout du grand nombre d'embryons ne donnant aucune plante et de ceux particulièrement productifs (10 plantes et plus), aussi bien pour les lignées que pour les hybrides.

En moyenne, les hybrides avec «CO158 » donnent moins de plantes que les hybrides avec "Al88». Contrairement à la lignée «A188 », « CO158 » est moins productive que ses hybrides.

\section{MORPHOLOGIE DES PLANTES RÉGÉNÉRÉES}

Parmi les plantes développées in vitro, certaines arrêtent leur croissance, d'autres n'émettent pas de racines. Seules les plantes complètes ont été repiquées ; leur reprise a été difficile puisque $23 \mathrm{p} .100$ d'entre elles survivent ; chez ces dernières (tabl. 7), on observe une forte réduction du nombre de feuilles et de la taille; des soies et des grains se développent quelquefois sur la partie mâle. Des épis complets ont été récoltés.
Un contrôle du nombre chromosomique fait sur les méristèmes racinaires de 35 de-ces plantes a montré que dans tous les cas $2 \mathrm{n}=20$.

\section{DISCUSSION ET CONCLUSION}

Au niveau de la culture in vitro, la moyenne des plantes produites par classe de taille d'embryon est nettement plus élevée entre 1 et $2,15 \mathrm{~mm}$, pour l'ensemble des lignées testées. BECKERT (1982), avec «A188», obtient la meilleure productivité en plantes pour des tailles très voisines d'embryons.

Cependant, nos valeurs moyennes en plantes produites par embryon morphogène sont plus élevées que celles trouvées par cet auteur, pour toute l'étendue des classes de taille. Cette différence semble provenir du mode de comptage des plantes ; toutefois, des résultats concordants sont observés en ce qui concerne l'hétérogénéité des embryons pour leur productivité en plantes, tant chez les lignées que chez les hybrides.

Différentes durées de culture préliminaire in vitro (lignée «A188» seulement) n'ont pas permis de déceler un effet du traitement, même pour des temps très courts, sur la proportion d'embryons morphogènes ; il semblerait toutefois que, dans nos expériences, la variabilité constatée soit due à un effet «plantemère ».

D'après des résultats obtenus par BECKERT (1982), la proportion d'embryons morphogènes n'est pas différente à l'intérieur d'une même classe de taille d'embryons issus de plusieurs plantes-mères.

L'emploi du 2,4D ou du pCA n'a pas mis en évidence un effet spécifique de ces auxines sur la régénération des lignées testées.

Les lignées ont pu être classées suivant leur aptitude à la régénération. "A188 " a montré de bonnes capacités et «F1412 » s'est montrée la plus faible ; plusieurs autres lignées sont intermédiaires entre ces extrêmes. Cette variabilité génétique dans l'aptitude à la régénération a été observée fréquemment chez diverses espèces, notamment chez les pétunias (DULIEU et al., 1983) et chez le mais (BECKERT \&

TABLEAU 7

Reprise et morphologie des plantes issues de culture in vitro.

Growth and morphology of plants originating from in vitro cultures.

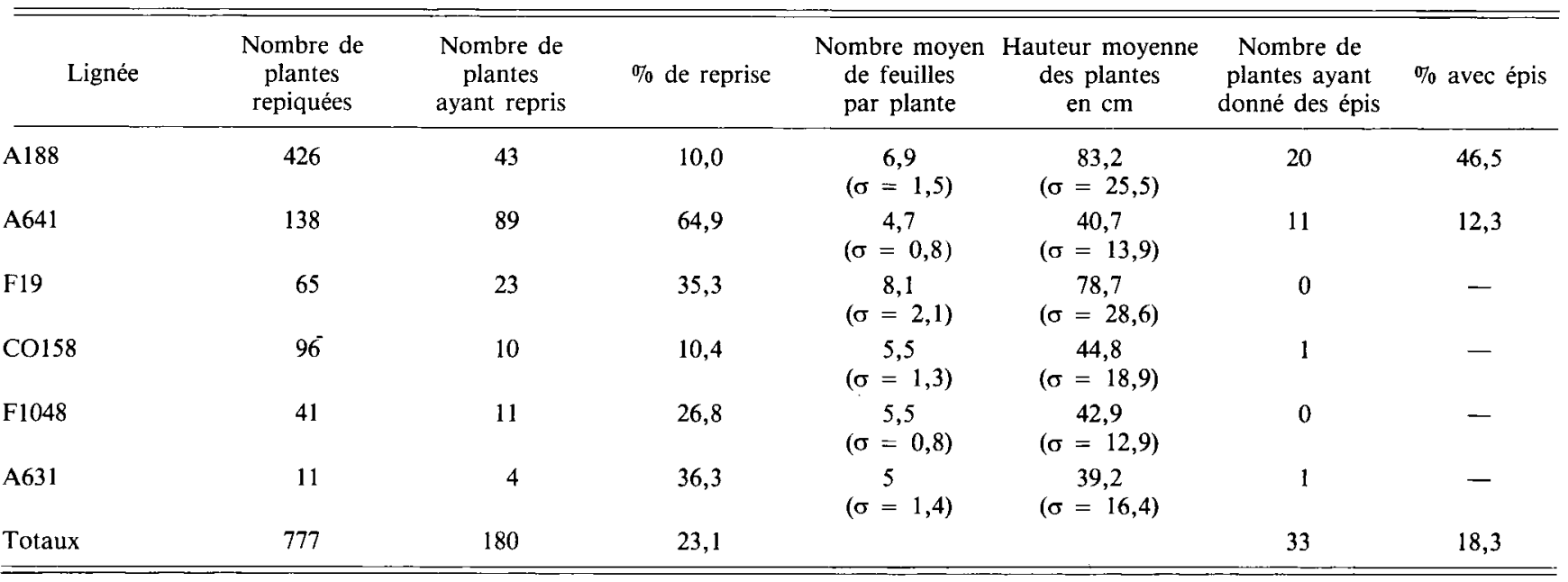


POllacseK, 1979 ; Beckert \& CaO Ming QING, 1984).

Les résultats de nos croisements réciproques ont montré que l'aptitude à la régénération se comporte comme un caractère dominant, notamment avec la lignée "A188 ». Cette dernière pourrait servir de géniteur pour améliorer les capacités de régénération de matériels considérés comme difficiles.

$\mathrm{La}$ régénération in vitro de plantes à partir d'embryons immatures chez le maïs paraît un phénomène complexe dont le succès dépend encore de nombreux facteurs non contrôlés.

Si l'étude des conditions de culture in vitro pour la callogenèse et la régénération a été surtout abordée, l'importance des conditions de culture des plantesmères sur les résultats recherchés n'a jusqu'à présent pas été souligné.

Une continuité expérimentale allant de la plante, cultivée in vivo dans des conditions strictement définies (température, énergie lumineuse, spectre...), à l'explant cultivé in vitro permettrait probablement une meilleure expression des capacités de chaque génotype (callogenèse, régénération) et devrait progressivement faciliter l'utilisation de ces génotypes pour des travaux de mutagenèse ou pour la culture de protoplastes.

Dans nos expériences, des plantes régénérées in vitro ont présenté in vivo des morphologies anormales ; ceci a été observé par divers auteurs : GREEN \&
Phillips (1975), Green (1977), Beckert \& PollaCSEK (1979). Ces observations sont à rapprocher de celles faites par MESSIAEN (1963) qui constate des anomalies morphologiques du même type après repiquage lorsque le stade végétatif est dépassé. GREEN (1977) et nous-mêmes avons observé sur ces plantes un nombre normal de chromosomes.

A partir de plantes régénérées in vitro BECKERT et al. (1983) ont, en choisissant des caractères agronomiques et morphologiques, analysé la variabilité génétique existante. Selon ces auteurs, la variabilité génétique de descendances de plantes obtenues in vitro est de très faible amplitude, sans commune mesure avec celle observée couramment chez le maïs.

Les diverses morphologies observées sont donc, pour une part importante, dues au passage in vitro qui perturbe le développement des plantes et ne sont généralement pas héréditaires.

Recu le 13 juillet 1984. Accepté le 22 février 1985.

\section{REMERCIEMENTS}

Je remercie MM. A. Cornu, H. Dulieu et D. Maizonnier (I.N.R.A., Dijon) pour l'aide apportée à l'élaboration de ce manuscrit.

\section{RÉFÉRENCES BIBLIOGRAPHIQUES}

Beckert M., 1982. Rôle du scutellum dans l'obtention de plantes néoformées in vitro chez le maïs. Agronomie, 2 (7), 612-615.

Beckert M., Cao Ming Quing, 1.984. Results of a diallel trial and a breeding experiment for in vitro aptitude. Theor. Appl. Genet., $68,247-251$.

Beckert M., Pollacsek M., 1979. Expression de la variabilité génétique du maîs (Zea mays L.) en clifférentes conditions de cultures de tissus. Ann. Amelior. Plant., 29, (5), 563-581.

Beckert M., Pollacsek M., Caenen M., 1983. Etude de la variabilité génétique obtenue chez le maïs après callogenèse et régénération de plantes in vitro. Agronomie, 3 (1), 9-18.

Cummings D. P., Green C. E., Stutheman D. D., 1976. Callus induction and plant regeneration in oats. Crop Sci., 16, 465-470.

Dale P. J., Deambrogio E. A., 1979. A comparaison of callus induction and plant regeneration from different explants of Hordeum vulgare. Z. Pflanzenphysiol., 94 (5), 65-77.

Dulieu H. L., Bruneau R., Pelletier A., 1983. Heritable difference in in vitro regenerability in Petunia, at the protoplast and the seed- ling stage. Poster proceedings, 236-237. 6th Int. Protoplast Simp. Ed. Birkhäuser Verlag, $366 \mathrm{p}$.

Gamborg O. L., Shyluk J. P., Brar J. P., Constabel F., 1977. Morphogenesis and plant regeneration from callus of immature embryos of Sorghum. Plant. Sci. Lett., 10, 67-74.

Green C. E., 1977. Prospects for crop improvement in the field of cell culture. Hortscience, 12, (2), 131-134.

Green C. E., 1979. Current status of cell and tissue culture methods in maize. Maize Genetic Workshop, University of Illinois. 1-18.

Green C. E., 1982. Somatic embryogenesis and plant regeneration from the friable callus of Zea mays. Proc. 5th Int. Cong. Plant Tissue and Cell culture. In E. Earle and Y. Demarly : "Plant tissue Culture ».

Green C. E., Phillips R. L., 1975. Plant regeneration from tissue culture of maize. Crop Sci., 15, 417-421.

Messiaen C. M., 1963. Physiologie du développement chez Zea mays $L$. Thèse Doct. Etat Univ. Aix-Marseille. Ann. Epiphyt., No Hors-Serie II, $74 \mathrm{p}$. 